ARTICLE

Received 1 Apr 2015 | Accepted 1 Sep 2015 | Published 30 Sep 2015

\title{
Ultralow-threshold multiphoton-pumped lasing from colloidal nanoplatelets in solution
}

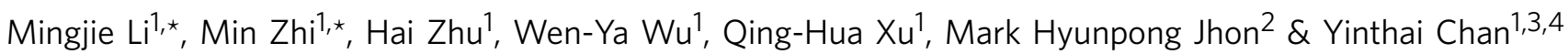

Although multiphoton-pumped lasing from a solution of chromophores is important in the emerging fields of nonlinear optofluidics and bio-photonics, conventionally used organic dyes are often rendered unsuitable because of relatively small multiphoton absorption crosssections and low photostability. Here, we demonstrate highly photostable, ultralow-threshold multiphoton-pumped biexcitonic lasing from a solution of colloidal $\mathrm{CdSe} / \mathrm{CdS}$ nanoplatelets within a cuvette-based Fabry-Pérot optical resonator. We find that colloidal nanoplatelets surprisingly exhibit an optimal lateral size that minimizes lasing threshold. These nanoplatelets possess very large gain cross-sections of $7.3 \times 10^{-14} \mathrm{~cm}^{2}$ and ultralow lasing thresholds of 1.2 and $4.3 \mathrm{~mJ} \mathrm{~cm}^{-2}$ under two-photon $\left(\lambda_{\mathrm{exc}}=800 \mathrm{~nm}\right)$ and three-photon $\left(\lambda_{\text {exc }}=1.3 \mu \mathrm{m}\right)$ excitation, respectively. The highly polarized emission from the nanoplatelet laser shows no significant photodegradation over $10^{7}$ laser shots. These findings constitute a more comprehensive understanding of the utility of colloidal semiconductor nanoparticles as the gain medium in high-performance frequency-upconversion liquid lasers.

\footnotetext{
${ }^{1}$ Department of Chemistry, National University of Singapore, 3 Science Drive 3, Singapore 117543, Singapore. ${ }^{2}$ Institute of High Performance Computing $A^{\star}$ STAR, 1 Fusionopolis Way, \#16-16 Connexis, Singapore 138632, Singapore. ${ }^{3}$ Institute of Materials Research \& Engineering A*STAR, 3 Research Link, Singapore 117602, Singapore. ${ }^{4}$ Microfluidics Systems Biology Lab, Institute of Molecular and Cell Biology A*STAR, 61 Biopolis Drive, Singapore 138673, Singapore. * These authors contributed equally to this work. Correspondence and requests for materials should be addressed to Y.T.C. (email: chmchany@nus.edu.sg).
} 
C olloidal semiconductor nanocrystals (NCs) are very attractive as optical gain media owing to their ease of fabrication, wide size-dependent colour tunability and spectrally narrow gain profiles ${ }^{1-5}$. Despite the first observation of optically pumped stimulated emission (SE) in a close-packed film of semiconductor NCs more than a decade ago ${ }^{1}$, only a very limited number of studies have documented the achievement of $\mathrm{SE}$ or lasing from NCs in solution so $\mathrm{far}^{6-8}$. Nevertheless, a solution-based gain medium can be highly desirable because of its flexibility of incorporation into optical cavities of any size and shape. For example, such a gain medium could be incorporated into optofluidic lasers ${ }^{9-11}$, which integrate microfluidics, a liquid gain medium and optical resonators within microchannels and which have already been utilized for sensitive bio/chemical intracavity detection ${ }^{12,13}$ and on-chip imaging ${ }^{14}$. Concurrently, significant efforts have been made in designing semiconductor NCs that can produce SE under excitation with a photon energy that is much lower than their bandgap through a multiphoton absorption process ${ }^{15-18}$. Emission from semiconductor NCs in solution obtained via multiphoton excitation can offer distinct advantages in the fields of nonlinear photonics and imaging in vivo that include deeper tissue penetration as well as less Rayleigh scattering, photodamage and photobleaching ${ }^{19-22}$. If these multiphoton-pumped (MPP) semiconductor NCs can be made to produce SE rather than spontaneous emission, they can be harnessed in the emerging field of optofluidic biolasers and can potentially improve the sensitivity and resolution of bio-imaging at the molecular, cellular and tissue level ${ }^{23-25}$.

Although MPP lasing has previously been realized using solutions of certain organic dyes ${ }^{26,27}$, semiconductor NCs can offer considerable advantages in terms of their relatively large multiphoton absorption cross-sections and high photostability ${ }^{28}$. However, to the best of our knowledge, neither MPP-amplified spontaneous emission (ASE) nor lasing has been observed from semiconductor NCs in solution, and only recently has threephoton-pumped SE been achieved in a close-packed film of $\mathrm{NCs}^{18}$. One major impediment is the extremely fast non-radiative Auger recombination of multiexcitons in semiconductor NCs or nanorods that prevents sustained population inversion and therefore optical gain ${ }^{1}$. For the achievement of SE, the SE build-up time, which is reciprocally related to the volume fraction of NCs within the gain medium, should be faster than the Augerdominated lifetime of the gain excited state. Understandably, it is very difficult to achieve lasing in solution given the low volume fraction of NCs and relatively small gain cross-section on a per $\mathrm{NC}$ basis (for example, $\sim 10^{-17} \mathrm{~cm}^{2}$ for $2.6 \mathrm{~nm}$ diameter CdSe $\mathrm{NCs}^{1}$ ). Furthermore, the threshold pump intensity for MPP SE is typically much higher than that for one-photon excitation ${ }^{17}$, making it challenging to prevent artifacts such as solvent boiling or evaporation within a practical device. Overcoming these obstacles requires semiconductor nanostructures with large multiphoton absorption cross-section and reduced multiexcitonic Auger recombination rates.

Recently, colloidal quasi-two-dimensional (2D) quantum wells, also known as semiconductor nanoplatelets (NPLs) have attracted much attention as a new class of solution-processable optical gain media since they retain many of the salient features of semiconductor NCs while also possessing high oscillator strengths, large absorption cross-sections and strongly suppressed Auger recombination rates ${ }^{29,30}$.

Herein, we demonstrate that two- and three-photon-pumped (2PP and 3PP) lasing from colloidal CdSe/CdS core/shell NPLs in solution can be achieved using a cuvette-based Fabry-Pérot cavity. Laser emission from the MPP NPL solution was highly photostable, thermally stable, directional and polarized. The concentration and lateral size of NPLs were found to have significant effects on the lasing threshold. The gain cross-section per NPL was determined to be a staggering $\sim 7.3 \times 10^{-14} \mathrm{~cm}^{2}$, and the lasing threshold pump fluence $\left(F_{\text {th }}\right)$ in solution was as low as $\sim 1.2$ and $\sim 4.3 \mathrm{~mJ} \mathrm{~cm}^{-2}$ for two- and three-photon excitation respectively. Remarkably, the lasing thresholds measured here are 3-10 times lower than those previously reported for MPP ASE from spherical semiconductor NCs in a closed-packed matrix despite the higher volume fraction ${ }^{18,31,32}$. These findings collectively highlight the potential of colloidal semiconductor NPLs as gain media for high-performance upconversion lasers, and provide guidelines for utilizing them in optofluidic light amplification and stimulated emission-based biophotonic techniques.

\section{Results}

Synthesis and characterization. Colloidal CdS/CdSe core/shell NPLs with different lateral dimensions used in this study were synthesized by growing two monolayers of CdS shells on either side of four monolayer thick CdSe NPLs using a wet-chemical atomic layer deposition technique ${ }^{29}$ as described in detail in the Supplementary Methods. Figure 1a shows representative transmission electron microscope (TEM) images of core/shell $\mathrm{CdSe} / \mathrm{CdS}$ NPLs with a thickness of $\sim 2.75 \mathrm{~nm}$, average edge dimensions of $\sim 15 \times 25 \mathrm{~nm}$ and a resulting lateral area of $\sim 390 \mathrm{~nm}^{2}$ (named as NPL390). Overcoating the CdSe NPLs with two monolayers of CdS resulted in the band-edge absorption red shifting from 513 to $613 \mathrm{~nm}$ while the spontaneous emission was shifted from 515 to $622 \mathrm{~nm}$ (Fig. 1b). These findings are similar to previous observations of CdSe/CdS core/shell NPLs ${ }^{29}$.

2PP and 3PP lasing from NPLs solution. To generate MPP lasing in solution, as-synthesized NPLs were ligand exchanged with and dispersed in excess 5-amino-1-pentanol (AP) at a concentration of $C_{0}=21.6 \mu \mathrm{moll}^{-1}(\mu \mathrm{M})$ (see Supplementary Note 1 for the determination of concentration). The mixture of NPLs in AP served as the liquid gain medium in a quartz cuvette with a $1-\mathrm{cm}$ wide and 1-mm-thick optical pathlength (Fig. 1c). Our choice of AP as the ligand and solvent was motivated by its good affinity for the surface of the NPLs and the excellent dispersibility and colloidal stability conferred even at very high NPL concentrations. The cuvette was transversely excited by a pulsed laser source with a $\sim 150 \mathrm{fs}$ pulse width and $1 \mathrm{kHz}$ repetition rate. The excitation beam was focused into a stripe using a cylindrical lens, and the output emission was collected from one of the side faces of the cuvette in a direction approximately normal to the incident pump beam as schematically shown in the inset of Fig. 1c. Here, the two parallel optical windows of the cuvette can act as mirrors, thereby providing optical feedback for lasing in a manner akin to a planar Fabry-Pérot etalon cavity. The optical pump wavelengths were 800 and $1,300 \mathrm{~nm}$ for two-photon and three-photon excitation, respectively. For $800 \mathrm{~nm}$ excitation below threshold on sample NPL390, a striped beam of red light could be seen on the cuvette but not on a screen placed to its side (Fig. 1c). At excitation intensities above threshold, an intense spot of light could be observed on the screen as shown in Fig. 1d, thus signifying the onset of 2PP lasing (see Supplementary Fig. 1 for a photograph illustrating 3PP lasing). The near- and far-field intensity distribution of the $2 \mathrm{PP}$ and $3 \mathrm{PP}$ lasing at a pump fluence of $1.5 F_{\text {th }}$ were also characterized, yielding divergence angles commensurate with previously reported cuvette-based lasers ${ }^{27}$ (Supplementary Fig. 2, Supplementary Note 2 and Supplementary Table 1).

Figure 1e shows the $2 \mathrm{PP}$ lasing spectra at different pump fluence above threshold, with the emission peak located at $\sim 634 \mathrm{~nm}$. At larger pump intensities, well-resolved, equally spaced resonant modes were observed. A similar periodic pattern 
a

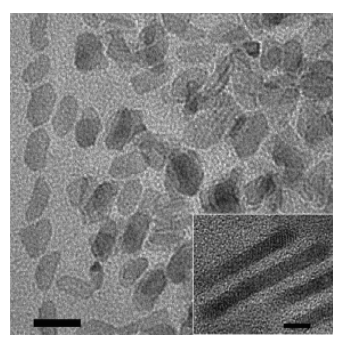

b

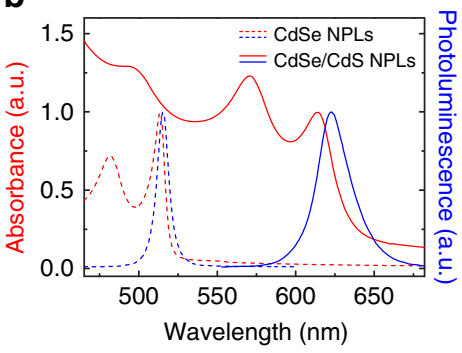

C

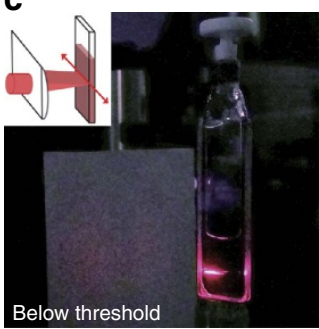

d

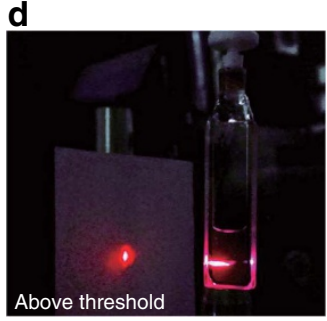

e

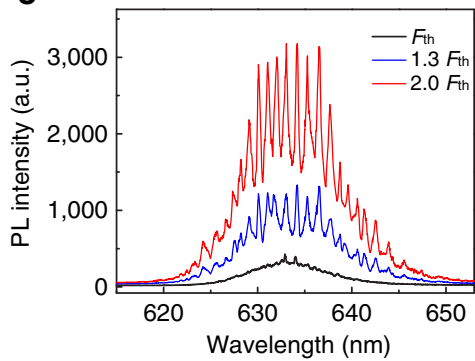

f

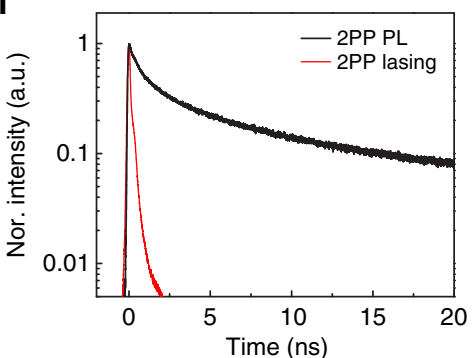

Figure 1 | MPP lasing from NPLs solution in a cuvette cell. (a) TEM image of sample NPL390 (scale bar, 25 nm). Inset shows the HRTEM of standing NPLs (scale bar, $5 \mathrm{~nm}$ ). (b) Absorption (red) and photoluminescence (blue) spectra of the CdSe (dash) and CdSe/CdS core/shell NPLs (solid). They are normalized at the first excitonic transitions. Photographs show the transversely excited cuvette filled with NPL solution under 800 nm femtosecond laser $(1 \mathrm{kHz}, 150 \mathrm{fs})$ excitation at the pump fluence $(\mathbf{c})$ below $\left(0.9 F_{\mathrm{th}}\right)$ and $(\mathbf{d})$ above lasing threshold $\left(1.3 F_{\mathrm{th}}\right) . F_{\text {th }}=1.2 \mathrm{~mJ} \mathrm{~cm}-2$. The distance

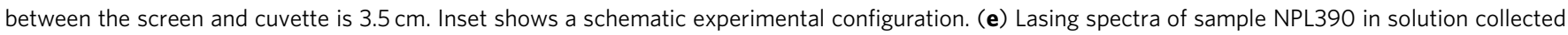
at the edge of cuvette under different pump fluence. (f) Fluorescence decay profiles of spontaneous emission (black) and 2PP lasing (red).

of lasing spectra was previously observed from a solution of Coumarin dye in a transversely excited cuvette ${ }^{33}$. Such spectral modulation was attributed to a Fabry-Pérot cavity formed by the front and back windows of the quartz cuvette. The wavelength spacing of two adjacent maxima is determined via the following relation $^{33}$ :

$$
\Delta \lambda=\frac{(\bar{\lambda})^{2}}{2 n^{\prime}\left|l_{\mathrm{f}}-l_{\mathrm{b}}\right|}
$$

where $\bar{\lambda}$ is the average lasing wavelength, $n$ ' is the refractive index of quartz, $l_{\mathrm{f}}$ and $l_{\mathrm{b}}$ are the thicknesses of the front and back windows of the cuvette. The value of $\left|l_{\mathrm{f}}-l_{\mathrm{b}}\right|$ was found to be $115 \pm 5 \mu \mathrm{m}$ by a digital micrometre. The calculated $\Delta \lambda$ of $1.1 \pm 0.1 \mathrm{~nm}$ agrees with the experimental spacing of $\sim 1.0 \mathrm{~nm}$ as seen in Fig. 1e, which further corroborates the fact that our cuvette functioned as a Fabry-Pérot cavity.

We also determined that the cuvette used in our experiments possessed a relatively high quality factor $(Q)$ of $\sim 1,200$ as calculated by $Q=\lambda / \delta \lambda$. Here $\lambda$ refers to the peak wavelength of the lasing transition while $\delta \lambda(\sim 0.54 \mathrm{~nm})$ is its corresponding fullwidth at half-maximum. The width of the entire set of peaks is about $10 \mathrm{~nm}$, and can likely be made narrower by reducing the size dispersity of the NPLs. In addition, the emission decay lifetime decreased sharply from $\sim 1.9 \mathrm{~ns}$ to a value approaching the instrument response time ( $\sim 50 \mathrm{ps})$ of our time-correlated singlephoton counting setup below and above threshold (Fig. 1f). It should be noted that during the course of the measurements, evaporation of the solvent or microscopic aggregation of the NPLs were not observed. Taken together, these findings indicate strongly that 2PP lasing from NPLs in solution was successfully achieved.

Effect of reabsorption on lasing. At the high NPL concentrations necessary to attain SE, it was found unexpectedly that the 2PP lasing peak was located at the higher energy side of its photoluminescence (PL) (Fig. 2a). In contrast, the one-photon pumped (1PP) lasing peak emerges from the lower energy side of its PL, as expected from a positive biexciton-binding energy $\left(E_{\mathrm{XX}}^{\mathrm{B}}\right)$ (ref. 30). It is worth noting that due to a considerable overlap of the absorption tail with the PL (Fig. 1b), as well as a relatively large penetration depth of the excitation stripe into the sample under MPP conditions, reabsorption of emission under multiphoton excitation ${ }^{34}$ can be very significant for high concentrations of NPLs. This in turn causes a red shift in the spontaneous emission, which as shown in Fig. 2a, can be as large as $\sim 16 \mathrm{~nm}$. Conversely, no obvious difference in PL spectra was observed from the low- and high-concentrated NPLs solution under one photon excitation because of its much shallower penetration depth. The 2PP lasing peak, however, has only a slight $\sim 3 \mathrm{~nm}$ red shift compared with the 1PP lasing peak because at the high excitation intensities required to achieve population inversion, the first excited state of most of the NPLs is filled and the corresponding absorption transition is bleached out. Therefore, the emergence of the 2PP lasing peak at the higher energy side of its 2PP PL spectrum is not due to the occurrence of a negative biexciton energy of interaction but rather the effect of reabsorption.

Characteristics of MPP biexcitonic lasing. The output energy per pulse versus pump fluence from NPL solution laser is given in the log-log plot in Fig. 2b, where the s-shaped profile suggests a distinct transition from 2PP PL to 2PP lasing and then saturation. For comparison, a linear plot of the same data is provided in the lower right figure inset, where a clear threshold behaviour is readily observed. The quadratic power dependence obtained in the low excitation regime is attributed to spontaneous emission via two-photon absorption (2PA). The transition to a quartic power dependence of the output energy with pump fluence, which is accompanied by a narrowing of the emission spectrum into a few sharp peaks as illustrated in Fig. 2a (red curve), indicates the onset of lasing. The fourth power dependence is consistent with the assumption that the origin of the optical gain is largely biexcitonic in nature since the NPLs are excited from 
a

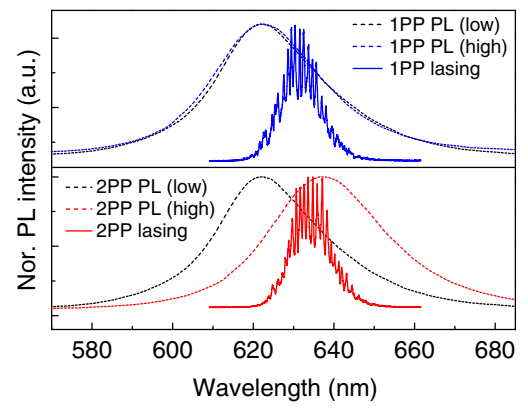

C

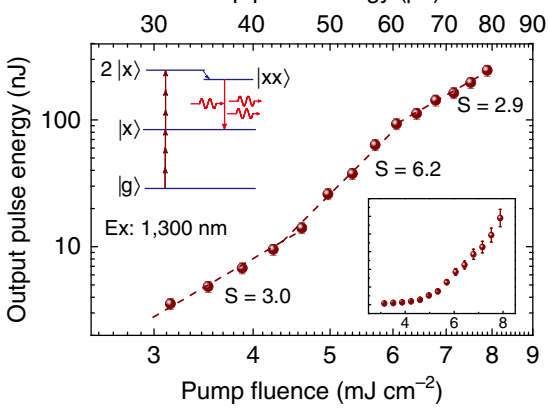

b

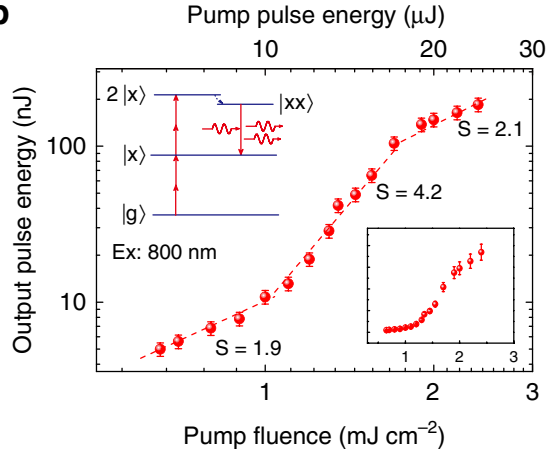

d

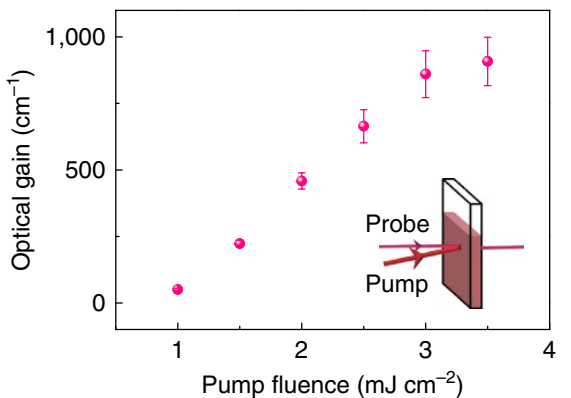

Figure 2 | Biexcitonic lasing and optical gain. (a) Normalized (Nor.) 1PP and 2PP PL (dashed line) and lasing (solid line) spectra for sample NPL390 in solution. Low and high refer to the NPL concentration of $\sim 0.001 C_{0}$ and $C_{0}$, respectively. The concentration for $1 \mathrm{PP}$ and $2 \mathrm{PP}$ lasing is $C_{0}$. The log-log plots of laser output energy versus pump fluence/energy together with the slopes (S) excited with (b) $800 \mathrm{~nm}$ and (c) 1,300 nm light for two- and three-photon excitation, respectively. Linear plots of laser output energy versus pump fluence are at the lower right insets of $\mathbf{b}$ and $\mathbf{c}$. (d) Optical single-pass gain versus pump fluence for sample NPL390 in solution with concentration of $C_{0}$. Inset shows the experimental configuration. The error bars were determined based on the standard deviation of four repeated acquisitions.

their ground to single exciton states and then subsequently to biexciton states (as shown schematically in the upper left inset of Fig. 2b). The power dependence returns to a quadratic slope at pump powers well above the lasing threshold, which is expected from gain saturation. $E_{\mathrm{XX}}^{\mathrm{B}}$ was determined by the energy difference $(\sim 30 \mathrm{meV})$ between the single-exciton emission peak at very low pump fluence and the fitted biexciton emission peak at high pump fluence of a 1PP solution of NPLs at low concentration (Supplementary Fig. 3 and Supplementary Note 3).

In the case of $3 \mathrm{PP}$ lasing, the output energy versus pump fluence plot shows a trend analogous to that of the $2 \mathrm{PP}$ experiment. Spontaneous emission at low excitation fluence yielded a cubic dependence of the output intensity on pump energy while the onset of lasing at higher fluence was accompanied by a sixth power dependence, as shown in Fig. 2c. As with the 2PP data, the fitted exponents to the output intensity under 3PP agrees with a biexciton gain model, as illustrated in the upper left inset of Fig. 2c. At large pump intensities, the relationship between output and input energy is again approximately cubic, signifying gain saturation. A corresponding linear plot is given in the lower right inset of Fig. $2 c$ to aid in visualizing clear threshold behaviour and the onset of optical amplification.

For sample NPL390 at a concentration of $C_{0}=21.6 \mu \mathrm{M}$ in AP, $F_{\text {th }}$ for $2 \mathrm{PP}$ lasing was as low as $\sim 1.2 \mathrm{~mJ} \mathrm{~cm}^{-2}$, which is about 5-10 times smaller than 2PP ASE from close-packed films of $\mathrm{CdZnS} / \mathrm{ZnS} \mathrm{NCs}^{31}$, and $\mathrm{CdSe} / \mathrm{CdS} / \mathrm{ZnS} \mathrm{NCs}^{32}$. Similarly, the threshold for 3PP lasing was determined to be $\sim 4.3 \mathrm{~mJ} \mathrm{~cm}^{-2}$, which is about $\sim 3$ times lower than that for 3PP ASE in closepacked films of $\mathrm{CdSe} / \mathrm{CdS} / \mathrm{ZnS} \mathrm{NCs}^{18}$. The average number of initially generated excitons per NPL $(<\mathrm{N}\rangle)$ can be calculated by using $<\mathrm{N}>_{2 \mathrm{PP}}=F^{2} \sigma_{2} \tau^{-1}$ and $<\mathrm{N}>_{3 \mathrm{PP}}=F^{3} \sigma_{3} \tau^{-2}$, where $F$ is the pump fluence, $\tau$ is the pulse duration, and $\sigma_{2}$ and $\sigma_{3}$ are the two-photon and three-photon absorption cross-sections measured by $\mathrm{Z}$-scan technique $\mathrm{e}^{35,36}$, respectively. The Z-scan curves (Supplementary Fig. 4) and detailed data processing for the extraction of $\sigma_{2}$ and $\sigma_{3}$ are given in the Supplementary Note 4. For the sample NPL390, $\sigma_{2}$ and $\sigma_{3}$ are about $7.9 \times 10^{-45} \mathrm{~cm}^{4} \mathrm{~s}$ per photon and $1.5 \times 10^{-75} \mathrm{~cm}^{6} \mathrm{~s}^{2}$ per photon ${ }^{2}$, these values are 2-4 orders of magnitude larger than that of previously reported semiconductor NCs and about 4 orders of magnitude larger than that of typical organic dyes $34,37,38$. On the basis of the threshold pump fluence, the values of $\langle\mathrm{N}\rangle_{2 \mathrm{PP} \text {-th }}$ and $\langle\mathrm{N}\rangle_{3 \mathrm{PP} \text {-th }}$ are calculated to be $\sim 1.2$ and $\sim 1.5$ for $2 \mathrm{PP}$ and $3 \mathrm{PP}$ lasing, respectively, which is consistent with what is known of NCs exhibiting biexcitonic gain ${ }^{1}$.

To estimate the energy conversion efficiency of our MPP cuvette-based NPL laser, the ratio of the output energy to the absorbed excitation energy was determined. As shown in Fig. 2b, at a pump energy level of $20 \mu \mathrm{J}$, the output energy per pulse of 2PP lasing is $\sim 150 \mathrm{~nJ}$. At this pump level, the attenuation ratio of the pump energy due to $2 \mathrm{PA}$ was measured to be 0.42 . Assuming that laser emission from both ends of the symmetric excitation stripe are identical, the overall energy conversion efficiency in the absence of absorption losses by unexcited regions of the gain solution (as observed in Fig. $1 \mathrm{~d}$ ) will be $\eta_{2 \text { pp-out }}=3.6 \%$. Similarly, for $3 \mathrm{PP}$ lasing, $\eta_{3 \mathrm{pp} \text {-out }}=1.2 \%$. Although efficiencies as large as $\sim 10 \%$ in cuvette-based dye lasers have recently been reported ${ }^{27}$, further improvements to the quantum yield or absorption crosssection of the NPLs should allow for a substantial increase in their lasing energy conversion efficiency.

Gain cross-section per NPL. The optical gain of a solution of NPLs, which provides some indication of lasing performance, was 
determined by pump-probe transmission measurements. Figure $2 \mathrm{~d}$ shows the measured single-pass gain $g$ for the sample NPL390 at different pump fluence under two-photon excitation (see Supplementary Note 5 for the details of the determination of $g$ from pump-probe measurements). It is seen that $g$ increases gradually and then reaches a maximum value $g_{\max }$ of $950 \pm 100 \mathrm{~cm}^{-1}$ at $\sim 3 \mathrm{~mJ} \mathrm{~cm}{ }^{-2}$ as a result of gain saturation. Under this high pump fluence, virtually all photoexcited NPLs undergo complete population inversion and the NPL solution reaches its maximum gain, which we denote as $g_{\max }$. The gain cross-section per NPL $\left(\gamma_{g}\right)$ can be obtained via the relation $\gamma_{g} \approx g_{\max } / C_{0}$ and was found to be $7.3 \times 10^{-14} \mathrm{~cm}^{2}$. For comparison, this value of $\gamma_{g}$ is about three orders of magnitude greater than that of one-photon excited $2.6 \mathrm{~nm}$ diameter CdSe (ref. 1) and $8.5 \mathrm{~nm}$ diameter $\mathrm{CdZnS} / \mathrm{ZnS} \mathrm{NCs}{ }^{8}$. Notably, the gain crosssection per unit volume (that is, $\sim 6.7 \times 10^{5} \mathrm{~cm}^{-1}$ ) is at least two orders of magnitude greater than these NCs, which may be attributed to the higher density of states available for SE in $\mathrm{NPLs}^{29}$

Minimum concentration of NPLs for lasing. The concentration of NCs in solution is a critical factor for determining the threshold pump fluence to achieve lasing. With the above obtained value of $\gamma_{g}$, the minimum concentration $C_{\text {min }}$ required to achieve lasing can be estimated. It is well known that threshold gain $\left(g_{\text {th }}\right)$ should be equal to the sum of the optical losses of the cavity. For our cuvette-based Fabry-Pérot cavity, we have the following relation ${ }^{39}$ :

$$
g_{\text {th }}=\alpha_{\text {int }}+\frac{1}{L} \ln \frac{1}{R}
$$

where $L(=0.1 \mathrm{~cm})$ is the cavity length (that is, the optical pathlength of the cuvette in Fig. 1c), $R(\sim 0.04)$ is the reflectivity between air and quartz. $\alpha_{\text {int }}$ is the internal loss coefficient per unit length primarily due to free carrier absorption of NPLs. The second term on the right-hand side of equation (2) represents the mirror loss which was calculated to be $\sim 32 \mathrm{~cm}^{-1}$. At the minimum concentration of NPLs required for lasing, the threshold gain should be equal to its maximum gain, therefore $g_{\text {th }}=\gamma_{g} C_{\text {min }}$. $\alpha_{\text {int }}$ can be neglected due to nearcomplete absorption bleaching of the band-edge under high pump fluence. Under these conditions, equation (2) reduces to $950 C_{\min } / C_{0}=32$, which yields a minimum concentration $C_{\min } \approx 0.03 C_{0}$.

The threshold pump fluence of 2PP lasing for different concentrations of sample NPL390 were also measured, and the results are plotted in Fig. 3a. It is readily observed that $F_{\text {th }}$ initially increases slowly as the concentration is reduced and then increases rapidly at concentrations below $\sim 0.4 C_{0}$. The lowest experimentally determined NPL concentration capable of achieving lasing was $\sim 0.1 C_{0}$ (that is, $2.16 \mu \mathrm{M}$ ) with a corresponding pump threshold of $\sim 10.6 \mathrm{~mJ} \mathrm{~cm}^{-2}$, which is in reasonable agreement with $C_{\min }$. It is possible that the experimental minimum concentration may be lower, however our use of AP as the solvent resulted in boiling at the higher pump intensities required to achieve lasing in sample concentrations lower than $0.1 C_{0}$.

Photostability and polarization. Figure $3 \mathrm{~b}$ illustrates the photostability of a MPP semiconductor NPL solution-based laser under continuous excitation with the $1 \mathrm{kHz}$ femtosecond pump source. No noticeable degradation in the 2PP and 3PP laser emission intensities was observed over $\sim 1 \times 10^{7}$ laser pulses (corresponding to $\sim 3 \mathrm{~h}$ of excitation) at a pump fluence approximately twice higher than that of the lasing threshold. While longer excitation periods were not explored, it should be mentioned that the number of laser pulses the solution sample was exposed to without significant degradation observed was already two orders of magnitude larger than the ASE from solutions of organic dyes ${ }^{40}$, and at least one order of magnitude larger than that from $3 \mathrm{PP}$ close-packed $\mathrm{CdSe} / \mathrm{CdS} / \mathrm{ZnS}$ films ${ }^{18}$. The excellent photostability afforded by CdSe/CdS NPLs in AP, and the absence of solvent evaporation within the pump intensity employed, provide further evidence that our choice of gain material and solvent provides a promising route to a robust, high-performance liquid gain medium. In addition, it is often advantageous for many applications if the laser output is linearly polarized. Figure $3 \mathrm{c}$ shows that the lasing above threshold from 2PP NPLs in the cuvette was strongly polarized normal to the plane of incidence (TE polarization) to an extent of about $90 \%$, which is comparable to dye lasers ${ }^{41}$. Notably, thermal stability measurements from $23-120^{\circ} \mathrm{C}$ showed little variation in terms of threshold or polarization (Supplementary Fig. 5 and Supplementary Note 6).

Optimum NPL size for lowest threshold. In addition to the number density effects analysed previously, the lateral dimensions of NPLs can also play a critical role in determining their lasing threshold. To explore the size-dependent behaviour of NPLs for low-threshold lasing, eight batches of NPLs with varying lateral dimensions of $\sim 9 \mathrm{~nm} \times 9.5 \mathrm{~nm}$ to $\sim 44 \mathrm{~nm} \times 44 \mathrm{~nm}$ (corresponding to areas of $\sim 85 \mathrm{~nm}^{2}$ to $2,000 \mathrm{~nm}^{2}$ ) but with the same thickness $(\sim 2.75 \mathrm{~nm}$, see Supplementary Fig. 6 for HRTEM images) were investigated. Representative TEM images of the NPLs of small and large sizes are depicted in Fig. 4a,b. For the
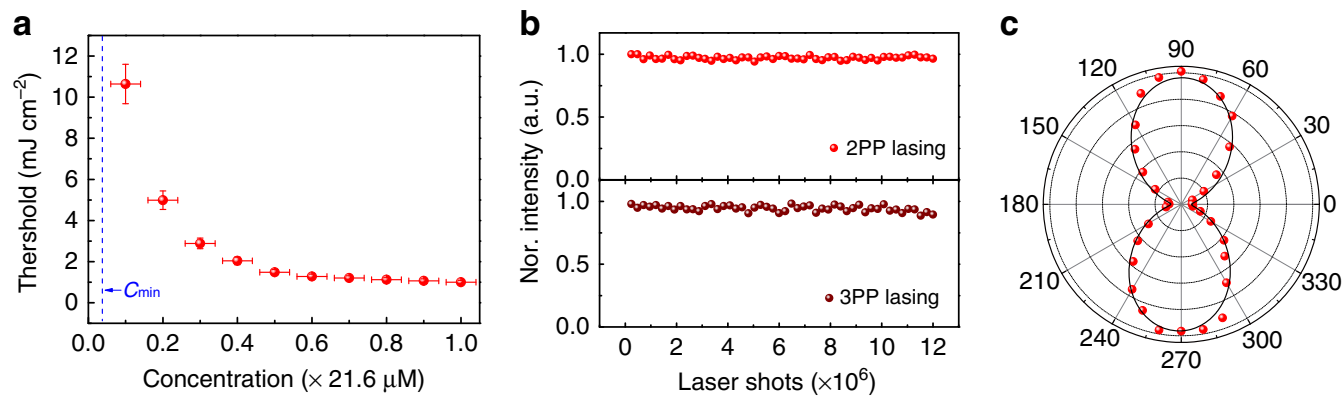

Figure 3 | Concentration effect, photostability and polarization of laser. (a) Threshold pump fluence of 2PP lasing for sample NPL390 in solution at different concentrations. The horizontal error bars were determined based on the uncertainty associated with obtaining the molar extinction coefficient of the NPLs and the vertical error bars were determined based on the standard deviation of four repeated acquisitions. (b) The normalized (Nor.) peak intensity of 2PP and 3PP lasing as a function of laser shots. (c) The laser emission above threshold as a function of angle $\theta$ between the plane of the polarizer in front of detector and a plane orthogonal to the cuvette surface. The solid black line is the fitting based on $\sin ^{2} \theta$. 
MPP lasing measurements, the concentrations of the different sized NPLs were adjusted to a constant value of $C_{0}=21.6 \mu \mathrm{M}$. It should be noted that the concentration of NPLs at relatively low concentration ranges can dramatically affect the threshold pump fluence whereas it is relatively insensitive to differentiate between high concentration values, as illustrated in Fig. 3a. Therefore, a high concentration of $C_{0}$ was used for all samples so that uncertainties in the measurement of $F_{\text {th }}$ as a result of errors in determining NPL concentration were minimal.

A clear trend was observed for the threshold pump fluence $F_{\text {th }}$ as a function of the lateral size of the NPLs for both 2PP (Fig. 4c) and 3PP lasing (Supplementary Fig. 7 ). In both cases, $F_{\text {th }}$ rapidly declines as the lateral size of the NPL increases, reaches a minimum, increases again and plateaus at the largest sizes measured. To gain deeper insight into the dependence of pump threshold on the lateral size of the NPLs, we determined the average number of excitons per NPL $\left(\langle\mathrm{N}\rangle_{\text {th }}\right)$ at the onset of 2PP lasing. This is an alternate measure for threshold that accounts for differences in the two-photon absorption crosssection for the various-sized NPLs. As shown in Fig. 4d, the dependence of $\langle\mathrm{N}\rangle_{\text {th }}$ on the lateral size of the NPLs mirrors the behaviour of the pump threshold: $\langle\mathrm{N}\rangle_{\text {th }}$ likewise exhibits a minimum at the same lateral size. This surprising threshold behaviour is not reconcilable with our general expectation that, for solutions at the same particle concentration but with different-sized NPLs, the larger-sized NPLs can achieve lower lasing threshold because of their large absorption cross-section (Table 1) and larger volume fractions. Further, the threshold behaviour cannot be attributed to variations in quantum yield because the deviation in quantum yield amongst the
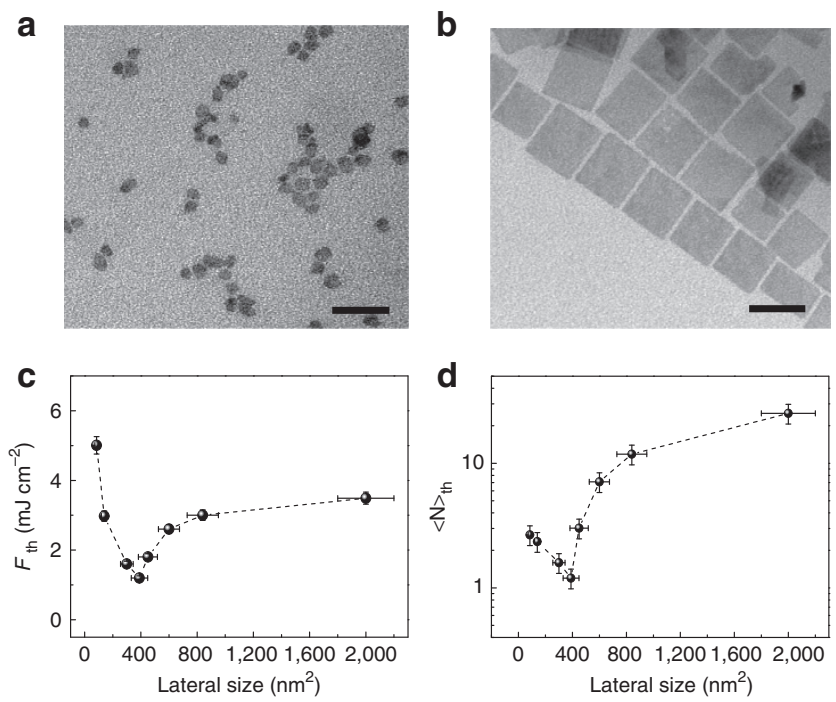

Figure 4 | Effect of lateral size on lasing threshold. TEM images of NPLs with average lateral size of (a) $85 \mathrm{~nm}^{2}$, (b) $2,000 \mathrm{~nm}^{2}$. Both scale bars, $50 \mathrm{~nm}$. (c) Threshold pump fluence and (d) initially generated of excitons per NPL at threshold pump fluence as a function of lateral sizes for 2PP lasing. The horizontal error bars were determined based on the lateral size distribution of the NPLs while the vertical error bars were determined based on the standard deviation of 4 repeated acquisitions. different-sized samples was small $(<3.9 \%$, see Table 1$)$ and does not correlate with the trends observed in Fig. $4 \mathrm{c}$,d.

We rationalize the minimum in the threshold by recognizing that quantum confinement can play a critical role in lasing. Given that the thicknesses of the different-sized NPLs were essentially the same, any differences in the extent of quantum confinement between different-sized NPLs should arise primarily from variations in their lateral dimensions. We estimate the radius of the biexciton to be $\sim 2.74$ times of the $2 \mathrm{D}$ exciton Bohr radius ${ }^{42}$. Taking the $2 \mathrm{D}$ Bohr radius $\alpha_{\mathrm{B}}$ in a CdSe NPL to be about $3.5 \mathrm{~nm}$ (ref. 43), we find that the biexciton radius should be about $\sim 9.6 \mathrm{~nm}$. This is much smaller than the linear size of the largest particles and so we conclude that the large particles are in the weak confinement regime. As the lateral size of an NPL is decreased, its behaviour will gradually cross over into the strong biexciton confinement limit as the lateral size approaches the $2 \mathrm{D}$ biexciton size. Experimental evidence of lateral quantum confinement with decreasing NPL size can be observed from the slight blue shift in core CdSe NPL emission, which is accompanied by a larger red shift after overcoating with CdS (Supplementary Fig. 8) as was previously observed in small CdSe nanoparticles coated with a shell of $\mathrm{ZnS}$ (ref. 44).

The transition to strong confinement is generally accepted to be accompanied by a sharpening of the density of states and subsequent narrowing of the gain spectra ${ }^{45}$. This is a significant effect. A density matrix theory of lasers can be used to show that the peak gain can be increased by an order of magnitude at the same carrier density by transitioning from a quantum well structure to a quantum box structure ${ }^{46}$. The increase in gain at a given carrier density is reflected by a lowering of the pump threshold required for lasing. We also note that $E_{\mathrm{XX}}^{\mathrm{B}}$ of our NPLs in the intermediate confinement regime is fairly small $(\sim 30 \mathrm{meV})$, on the order of $k_{\mathrm{B}} T$. This binding energy is expected to decrease with reducing the quantum confinement ${ }^{46}$. This means that $E_{\mathrm{XX}}^{\mathrm{B}}$ for larger NPLs may drop below $k_{\mathrm{B}} T$, allowing for biexcitons to dissociate into uncorrelated excitons. In addition, the internal loss is expected to be higher for larger sized NPLs due an increase in free carrier absorption and scattering. These considerations collectively indicate that by reducing lateral size, the threshold number of excitons per NPL will be reduced, in agreement with our observations for NPLs with lateral areas of $\sim 400-2,000 \mathrm{~nm}^{2}$.

However, as the NPL size decreases further, the increased quantum confinement will inevitably lead to faster Auger recombination, which is known to progress at a rate inversely proportional to the volume of the particle for simple, spherical single component quantum dots ${ }^{1}$. Auger recombination rates will diverge and cause non-radiative recombination to eventually dominate carrier lifetime. We interpret the increasing $F_{\text {th }}$ for very small NPLs $\left(<400 \mathrm{~nm}^{2}\right)$ to reflect the stronger pumping required to compensate for increased Auger recombination. The optimum lateral area of NPLs for lowest lasing threshold thus corresponds to a size where the competing size effects of density of states, $E_{\mathrm{XX}}^{\mathrm{B}}$, internal loss and non-radiative Auger recombination are balanced.

\section{Discussion}

In summary, low threshold two-photon $\left(\lambda_{\mathrm{exc}}=800 \mathrm{~nm}\right)$ and three-photon $\left(\lambda_{\mathrm{exc}}=1,300 \mathrm{~nm}\right)$-pumped red lasing were

\begin{tabular}{|lccccccc}
\hline \multicolumn{6}{l}{ Table 1 I Summary of quantum yield and 2PA and 3PA cross-sections of NPLs with different average lateral sizes. } \\
Lateral size $\left(\mathrm{nm}^{2}\right)$ & 85 & 140 & 300 & 390 & 450 & 600 & 840 \\
Quantum yield (\%) & 24 & 17 & 24 & 26 & 23 & 18 & 20 \\
$\sigma_{2}\left(\times 10^{-45} \mathrm{~cm}^{4}\right.$ s per photon) & 1.3 & 2.8 & 6.0 & 7.9 & 7.4 & 8.9 & 12.5 \\
$\sigma_{3}\left(\times 10^{-75} \mathrm{~cm}^{6} \mathrm{~s}^{2}\right.$ per photon $\left.{ }^{2}\right)$ & 0.5 & 0.7 & 1.2 & 1.5 & 1.8 & 3.0 & 3.9 \\
\hline
\end{tabular}


successfully demonstrated in colloidal CdSe/CdS core/shell NPLs dispersed in 5-amino-1-pentanol within a cuvette-based optical cavity. The laser emission was found to be extremely photostable, enduring $>10^{7}$ laser shots without photodegradation or solvent evaporation. This is advantageous to colloidal semiconductor NC-based solid state lasers where a fixed subpopulation of particles are constantly excited. Among eight batches of NPLs with average lateral sizes ranging from $\sim 85$ to $2,000 \mathrm{~nm}^{2}$, NPLs with a lateral size of $\sim 390 \mathrm{~nm}^{2}$ (NPL390) which is close to the 2D biexciton size were found to exhibit the lowest threshold pump fluence and $<\mathrm{N}>$ required to achieve the lasing. For these NPLs, the lasing thresholds were extremely low (for example, $\sim 1.2$ and $4.3 \mathrm{~mJ} \mathrm{~cm}^{-2}$ per pulse under two- and three-photon excitation for NPLs solution with the concentration of $21.6 \mu \mathrm{M}$, respectively) and was mainly attributed to an increased density of states that resulted from increased lateral quantum confinement. The maximum optical gain of the solution of sample NPL390 measured by pump-probe technique was relatively large at $\sim 950 \mathrm{~cm}^{-1}$. The magnitude of the deduced gain cross-section was determined to be $\sim 10^{-14} \mathrm{~cm}^{2}$, which is three orders of magnitude larger than those reported for colloidal $\mathrm{NCs}^{1,8}$. In addition, it was found that the threshold pump fluence increased slowly and then rapidly as the concentration of NPLs in the sample was reduced. Accordingly, a judicious choice of NPL size and concentration should yield the best performance to material consumption ratio.

Our findings provide guidelines for designing low threshold solution-based NPL lasers, and should also be extendable to their solid state counterparts. Photo- and thermally stable, multiphoton pumped solution-based colloidal NPL gain media are good candidates for frequency upconversion optofluidic lasers. This colloidal gain media opens new possibilities in nanolasing. For example, our system lends itself to integration into plasmonic waveguides that could allow for cavity-free lasing using the stopped-light concept ${ }^{47}$. Because the gain medium in a solutionbased system can be changed on-the-fly, it is possible to engineer its properties independently of device-level properties such as optical confinement ${ }^{48}$. Overcoming the challenge of modifying the surface of NPLs to make them water dispersible and biocompatible while still preserving their desirable lasing properties will undoubtedly expand their utility as nonlinear optical biosensors.

\section{Methods}

Synthesis of CdSe/CdS nanoplatelets. Core/Shell CdSe/CdS NPLs with different lateral dimensions were synthesized according to previous $\operatorname{method~}^{29}$ with slight modifications (see Supplementary Methods for details). Briefly, four monolayer thick CdSe core NPLs of various lateral sizes were overcoated with CdS by depositing alternating atomic layers of $\mathrm{Cd}$ and $\mathrm{S}$ until about two monolayers thick CdS shells were obtained. The as-synthesized core/shell CdSe/CdS NPLs were dissolved in $\mathrm{N}$-methylformamide and then processed via two cycles of precipitation and re-dispersion in ethanol and $\mathrm{N}$-methylformamide. The resulting particles were subsequently dried under nitrogen and then dispersed in 1-amino-5-pentanol to yield concentrated but aggregation-free solutions of colloidal NPLs.

Optical characterization. PL quantum yield of NPLs in AP solution was measured using a Horiba Fluorolog-3 fluorescence spectrometer equipped with a Quanta- QUOTE integrating sphere. The excitation source for the lasing, time-resolved PL, pump-probe and Z-scan measurements was a femtosecond Ti-sapphire laser system (Spectra-Physics) with a repetition rate of $1 \mathrm{kHz}$ and a pulse duration of $\sim 150 \mathrm{fs}$. The wavelength of the pump laser was tuned by an optical parametric amplifier (Light conversion, TOPAS). For the optical pumped lasing experiments, the laser pulses were focused by a cylindrical lens (with focal length $=20 \mathrm{~cm}$ ) into a stripe with a spot size of $\sim 5 \mathrm{~mm} \times 0.2 \mathrm{~mm}$ (see Supplementary Fig. 9 for the pump beam profile) onto the surface of a cuvette whose optical pathlength was $1 \mathrm{~mm}$. The direction of the pump laser was normal to the surface of the cuvette window. The emission was collected from one of the edges of the cuvette by an optical fibre coupled to a spectrometer (Horiba iH320) and detected by a CCD camera (Synapse, Horiba) or a power metre (Fieldmate, Coherent) with a high-sensitivity optical sensor (OP-2 Vis, Coherent) used for measuring emission energy. Emission lifetimes were measured using a timecorrelated single-photon counting module (PicoQuant PicoHarp 300). For the Z-scan measurements, the cuvette containing the solution of NPLs was mounted on a linear delay stage and moved along a direction parallel to the propagation of the pump beam over the focal length of the lens (focal length $=20 \mathrm{~cm}$ ). Subsequently, the transmitted light was collected by a detector without any aperture placed in front of it. For pump-probe measurements, the probe beam was generated by focusing $800 \mathrm{~nm}$ wavelength laser pulses into a $1 \mathrm{~mm}$ sapphire plate in order to generate white-light continuum. The probe beam with a focused spot size of $\sim 20 \mu \mathrm{m}$ in diameter was allowed to vertically pass through a solution of NPLs kept in a very thin cuvette (optical pathlength $=200 \mu \mathrm{m}$ ) and the pump pulse with a focused spot size of $\sim 50 \mu \mathrm{m}$ in diameter was overlapped with the probe pulse. The angle between the probe and pump beams was $\sim 30$ degrees. The intensity of the transmitted probe pulse at the wavelength of the NPL sample lasing peak $(\sim 634 \mathrm{~nm})$ passing through a monochromator (MicroHR, Horiba) was measured by a lock-in-amplifier (SR830, Stanford Research Systems).

\section{References}

1. Klimov, V. I. et al. Optical gain and stimulated emission in nanocrystal quantum dots. Science 290, 314 (2000).

2. Sundar, V. C. et al. Soft-lithographically embossed multilayered distributedfeedback nanocrystal lasers. Adv. Mater. 16, 2137-2141 (2004).

3. Snee, P. T., Chan, Y., Nocera, D. G. \& Bawendi, M. G. Whispering-gallerymode lasing from a semiconductor nanocrystal/microsphere resonator composite. Adv. Mater. 17, 1131-1136 (2005).

4. Klimov, V. I. et al. Single-exciton optical gain in semiconductor nanocrystals Nature 447, 441-446 (2007).

5. Liao, Y., Xing, G., Mishra, N., Sum, T. Z. \& Chan, Y. Low threshold, amplified spontaneous emission from core-seeded semiconductor nanotetrapods incorporated into a sol-gel matrix. Adv. Mater. 24, OP159-OP164 (2012).

6. Kazes, M., Lewis, D. Y., Ebenstein, Y., Mokari, T. \& Banin, U. Lasing from semiconductor quantum rods in a cylindrical microcavity. Adv. Mater. 14, 317-321 (2002).

7. Schafer, J. et al. Quantum dot microdrop laser. Nano Lett. 8, 1709-1712 (2008)

8. Wang, Y. et al. Blue liquid lasers from solution of $\mathrm{CdZnS} / \mathrm{ZnS}$ ternary alloy quantum dots with quasi-continuous pumping. Adv. Mater. 1, 169-175 (2015)

9. Monat, C., Domachuk, P. \& Eggleton, B. J. Integrated optofluidics: a new river of light. Nat. Photon 1, 106-114 (2007).

10. Psaltis, D., Quake, S. R. \& Yang, C. Developing optofluidic technology through the fusion of microfluidics and optics. Nature 442, 381-386 (2006).

11. Fan, X. \& White, I. M. Optofluidic microsystems for chemical and biological analysis. Nat. Photon 5, 591-597 (2011).

12. Galas, J. C., Peroz, C., Kou, Q. \& Chen, Y. Microfluidic dye laser intracavity absorption. Appl. Phys. Lett. 89, 224101 (2006).

13. Shao, H., Wang, W., Lana, S. E. \& Lear, K. L. Optofluidic intracavity spectroscopy of canine lymphoma and lymphocytes. IEEE Photon. Tech. Lett. 20, 493-495 (2008).

14. Cui, X. et al. Lensless high-resolution on-chip optofluidic microscopes for Caenorhabditis elegans and cell imaging. Proc. Natl Acad. Sci. USA 105, 10670-10675 (2008).

15. Jasieniak, J. J. et al. Highly efficient amplified stimulated emission from CdSe-CdS-ZnS quantum dot doped waveguides with two-photon infrared optical pumping. Adv. Mater. 20, 69-73 (2008).

16. Todescato, F. et al. Soft-lithographed up-converted distributed feedback visible lasers based on CdSe-CdZnS-ZnS quantum dots. Adv. Funct. Mater. 22, 337-344 (2012).

17. Xing, G. et al. Ultralow-threshold two-photon pumped amplified spontaneous emission and lasing from seeded CdSe/CdS nanorod heterostructures. ACS Nano 6, 10835-10844 (2012).

18. Wang, Y. et al. Stimulated emission and lasing from CdSe/CdS/ZnS core-multi-shell quantum dots by simultaneous three-photon absorption. $A d v$. Mater. 26, 2954-2961 (2014).

19. Zipfel, W. R., Williams, R. M. \& Webb, W. W. Nonlinear magic: multiphoton microscopy in the biosciences. Nat. Biotechnol. 21, 1369-1377 (2003).

20. Helmchen, F. \& Denk, W. Deep tissue two-photon microscopy. Nat. Methods 2, 932-940 (2005)

21. Hoover, E. E. \& Squier, J. A. Advances in multiphoton microscopy technology. Nat. Photon 7, 93-101 (2013).

22. Guo, L. \& Wong, M. S. Multiphoton excited fluorescent materials for frequency upconversion emission and fluorescent probes. Adv. Mater. 26, 5400-5428 (2014).

23. Sonnefraud, Y. et al. Experimental proof of concept of nanoparticle-assisted STED. Nano Lett. 14, 4449-4453 (2014).

24. Hein, B., Willig, K. I. \& Hell, S. W. Stimulated emission depletion (STED) nanoscopy of a fluorescent protein-labeled organelle inside a living cell. Proc. Natl Acad. Sci. USA 105, 14271-14276 (2008).

25. Min, W. et al. Imaging chromophores with undetectable fluorescence by stimulated emission microscopy. Nature 461, 1105-1109 (2009). 
26. He, G. S., Markowicz, P. P., Lin, T. C. \& Prasad, P. N. Observation of stimulated emission by direct three-photon excitation. Nature 415, 767-770 (2002).

27. Zheng, Q. et al. Frequency-upconverted stimulated emission by simultaneous five-photon absorption. Nat. Photon 7, 234-239 (2013).

28. Resch-Genger, U., Grabolle, M., Cavaliere-Jaricot, S., Nitschke, R. \& Nann, T. et al. Quantum dots versus organic dyes as fluorescent labels. Nat. Methods 5, 763-775 (2008).

29. She, C. et al. Low-threshold stimulated emission using colloidal quantum wells. Nano Lett. 14, 2772-2777 (2014).

30. Grim, J. Q. et al. Continuous-wave biexciton lasing at room temperature using solution-processed quantum wells. Nat. Nanotechnol. 9, 891-895 (2014).

31. Guzelturk, B., Kelestemur, Y., Akgul, M. Z., Sharma, V. K. \& Demir, H. V. Ultralow threshold one-photon-and two-photon-pumped optical gain media of blue-emitting colloidal quantum dot films. J. Phys. Chem. Lett. 5, 2214-2218 (2014).

32. Zhang, C. et al. Two-photon-pumped lasing from colloidal nanocrystal quantum dots. Opt. Lett. 33, 2437-2439 (2008).

33. Nedumpara, R. J. et al. Study of solvent effect in laser emission from Coumarin 540 dye solution. Appl. Opt. 46, 4786-4792 (2007).

34. He, G. S. et al. Multi-photon excitation properties of CdSe quantum dots solutions and optical limiting behavior in infrared range. Opt. Exp. 15, 12818-12833 (2007).

35. Xing, G. et al. Enhanced tunability of the multiphoton absorption cross-section in seeded CdSe/CdS nanorod heterostructures. Appl. Phys. Lett. 97, 061112 (2010).

36. Xing, G. et al. Three-photon absorption in seeded CdSe/CdS nanorod heterostructures. J. Phys. Chem. C 115, 17711-17716 (2011).

37. $\mathrm{Pu}, \mathrm{S}$. C. et al. The Empirical correlation between size and two-photon absorption cross section of CdSe and CdTe quantum dots. Small 2, 1308-1313 (2006).

38. Xu, C. \& Webb, W. W. Measurement of two-photon excitation cross sections of molecular fluorophores with data from 690 to $1050 \mathrm{~nm}$. J. Opt. Soc. Am. B 13, 481-491 (1996).

39. Bass, M., Van Stryland, E. W., Williams, D. R. \& Wolfe, W. L. Handbook of Optics-Volume I Fundamentals, Techniques, and Design 2nd ed. (McGraw-Hill, 1995).

40. Howell, B. F. \& Kuzyk, M. G. Lasing action and photodegradation of disperse orange 11 dye in liquid solution. Appl. Phys. Lett. 85, 1901 (2004).

41. Nagata, I. \& Nakaya, T. Polarization of dye laser light. J. Phys. D: Appl. Phys. 6, 1870 (1973).

42. Singh, J., Birkedal, D., Lyssenko, V. G. \& Hvam, J. M. Binding energy of two-dimensional biexcitons. Phys. Rev. B 53, 15909-15913 (1996).

43. Tessier, M. D., Javaux, C., Maksimovic, I., Loriette, V. \& Dubertret, B. Spectroscopy of single CdSe nanoplatelets. ACS Nano 6, 6751-6758 (2012).

44. Dabbousi, B. O. et al. (CdSe) ZnS core-shell quantum dots: synthesis and characterization of a size series of highly luminescent nanocrystallites. J. Phys. Chem. B 101, 9463-9475 (1997).
45. Asada, M., Miyamoto, Y. \& Suematsu, Y. Gain and the threshold of three-dimensional quantum-box lasers. IEEE J. Quantum Electron. 22, 1915-1921 (1986).

46. Kang, K. I. et al. Confinement-enhanced biexciton binding energy in semiconductor quantum dots. Phys. Rev. B 48, 15449 (1993).

47. Pickering, T., Hamm, J. M., Page, A. F., Wuestner, S. \& Hess, O. Cavity-free plasmonic nanolasing enabled by dispersionless stopped light. Nat. Commun. 5, 4927 (2014).

48. Sidiropoulos, T. P. et al. Ultrafast plasmonic nanowire lasers near the surface plasmon frequency. Nat. Phys. 10, 870-876 (2014).

\section{Acknowledgements}

This work was supported by National Research Foundation (NRF) Competitive Research Programme (NRF-CRP8-2011-07), the NRF Singapore-Berkeley Research Initiative for Sustainable Energy (SinBerRISE) CREATE Programme and a Visiting Investigator Program grant from Joint Council Office, $A^{\star}$ STAR (project 1235e00049).

\section{Author contributions}

M.L. designed, carried out the lasing measurements and analysed the data. M.Z synthesized and characterized the materials. W.-Y.W. assisted in the HRTEM characterization. H.Z. and Q.-H.X. assisted in the pump-probe and Z-scan measurements. M.L., Y.C. and M.H.J. co-wrote the manuscript. All authors discussed the results and commented on the manuscript.

\section{Additional information}

Supplementary Information accompanies this paper at http://www.nature.com/ naturecommunications

Competing financial interests: The authors declare no competing financial interests.

Reprints and permission information is available online at http://npg.nature.com/ reprintsandpermissions/

How to cite this article: Li, M. et al. Ultralow-threshold multiphoton-pumped lasing from colloidal nanoplatelets in solution. Nat. Commun. 6:8513

doi: $10.1038 /$ ncomms9513 (2015)

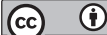

This work is licensed under a Creative Commons Attribution 4.0 International License. The images or other third party material in this article are included in the article's Creative Commons license, unless indicated otherwise in the credit line; if the material is not included under the Creative Commons license, users will need to obtain permission from the license holder to reproduce the material. To view a copy of this license, visit http://creativecommons.org/licenses/by/4.0/ 